Journal of Computer Science 6 (7): 706-715, 2010

ISSN 1549-3636

(C) 2010 Science Publications

\title{
Advances in Electromyogram Signal Classification to Improve the Quality of Life for the Disabled and Aged People
}

\author{
Md. R. Ahsan, Muhammad I. Ibrahimy and Othman O. Khalifa \\ Department of Electrical and Computer Engineering, International Islamic University Malaysia, \\ Gombak, 53100 Kuala Lumpur, Malaysia
}

\begin{abstract}
Problem statement: The social demands for the Quality Of Life (QOL) are increasing with the exponentially expanding silver generation. To improve the QOL of the disabled and elderly people, robotic researchers and biomedical engineers have been trying to combine their techniques into the rehabilitation systems. Various biomedical signals (biosignals) acquired from a specialized tissue, organ, or cell system like the nervous system are the driving force for the entire system. Examples of biosignals include Electro-Encephalogram (EEG), Electrooculogram (EOG), Electroneurogram (ENG) and (EMG). Approach: Among the biosignals, the research on EMG signal processing and controlling is currently expanding in various directions. EMG signal based research is ongoing for the development of simple, robust, user friendly, efficient interfacing devices/systems for the disabled. The advancement can be observed in the area of robotic devices, prosthesis limb, exoskeleton, wearable computer, I/O for virtual reality games and physical exercise equipments. An EMG signal based graphical controller or interfacing system enables the physically disabled to use word processing programs, other personal computer software and internet. Results: Depending on the application, the acquired and processed signals need to be classified for interpreting into mechanical force or machine/computer command. Conclusion: This study focused on the advances and improvements on different methodologies used for EMG signal classification with their efficiency, flexibility and applications. This review will be beneficial to the EMG signal researchers as a reference and comparison study of EMG classifier. For the development of robust, flexible and efficient applications, this study opened a pathway to the researchers in performing future comparative studies between different EMG classification methods.
\end{abstract}

Key words: QOL, EMG, HCI, neural network, fuzzy logic, Hidden Markov Model (HMM), Independent Component Analysis (ICA)

\section{INTRODUCTION}

With the advance of modern medical science, the percentage of elderly people is increasing day by day with fewer children. In Japan, the ratio of old-people aged 65 or more years is expected to be $20 \%$ by the year 2020 (Itou et al., 2001). More than 400,000 individuals are suffering in spinal cord injury or spinal dysfunction in USA and every year about 7800 injuries is adding (National Spinal Cord Injury Association, 1996). Additionally, there are large numbers of amputees, deaf people and other type of physically disabled people exists in our society. For the coming aging society, the welfare engineering section is trying their best to help the disabled (unable to walk) and oldpeople by developing better technical assistive devices. Today's advanced muscular sensing and processing technologies provide us Electromyogram (EMG) signal, which measures electrical potentials generated by muscle cells thus provide muscle activity. The measuring of EMG signals can be acquired either directly from within the muscle (termed as invasive EMG) or from surface of the skin above a muscle (termed as surface EMG or sEMG). Researchers are using EMG signals to diagnose muscular diseases, asses progress in muscular rehabilitation for the ever increasing societal recognition of the needs of people with physical disabilities. In addition these signals are also used for the controlling of prosthetics, computers as well as robotic devices. In the past three decades, advancement of EMG based control has received a great deal of attention for its application focused on rehabilitation and human-computer interfaces. EMG signal classification is the challenging part prior to interpreting it in corresponding control command. Large variations in EMG features make it difficult for pattern recognition. This is because the EMG signals have different signatures depending on age, muscle

Corresponding Author: Md. R. Ahsan, Department of Electrical and Computer Engineering,

International Islamic University Malaysia, Gombak, 53100 Kuala Lumpur, Malaysia 
development, motor unit paths, skin-fat layer and gesture style. Especially, it is difficult to extract useful features from the residual muscles of an amputee or disabled. So far, many researches proposed many kinds of EMG classification methodologies with satisfactory recognition performance.

The main focus of this study is to investigate by highlighting the different approaches and methodologies for the classification of EMG signals mainly in the field of Human Computer Interface (HCI) and prosthetics. This study firstly follows the classification techniques used for HCI with their relative applications, development and advantages/disadvantages. Afterwards, the review carries with EMG classification methodologies employed in the field of prosthetics with their gradual development and major characteristics.

EMG signal classification methodologies for HCI: Many of aged, disabled, bedridden, injured persons and the therapists who treat them, have lack of experience with computers and technology in general. Thus, to accommodate these types of users, the HCI of course intuitive, similar to the way computer mouse actions. HCI is transparent, supportive alternative means of rehabilitation rather than the focus of the patient and clinician. The first research on a human interface based on the EMG signal was initiated by Wiener (1948) and since then, basic researches on and practical uses of this concept have steadily progressed. Some Artificial Intelligence (AI) techniques mainly based on neural networks have been proposed for processing and discriminating EMG signal. Neural network is a computational structure which is evolved from mathematical models of biological neurons and neural processing. During recent years, neural networks have become a useful tool for the categorization of multivariate data. Even some of the cases, the Neural network combined with other AI e.g., Fuzzy, Hidden Markov Model (HMM), Bayes yields very good performance.

Artificial intelligence methods: Neural networks are very much popular nowadays because of their ability to imitate some of the brain's creative processes that cannot be populated by existing mathematical or logical methods. The incredible capabilities in the brain come up from its massive interconnected networks in between neurons. These networks can incrementally analyze and process information transmitted from the external or its internal environment and finally, a robust internal representation of the external phenomena has been outputted. This is termed as learning and the brain either can be trained or left alone for learning automatically. The same thing followed by artificial neural networks, where interconnected neurons organized into a network and each neuron processes data locally using the concepts of learning in the brain. Neural networks perform a variety of tasks, including prediction or function approximation, pattern classification, clustering and forecasting (Jain et al., 1996). The Fig. 1 represents the complex classification tasks by neural networks.

A real-time computer control system proposed by Putnam and Knapp (1993). The pattern recognition of the EMG signal from user's gestures was based on neural network. In the proposed system, there are two modes of communication which are derived from the EMG. In the first mode, a continuous control signal proportional to muscular exertion that controls the computer software objects such as sliders or scroll bars. The gesture recognition is second communication mode which allows the computer for making discrete choices such as menu selections or slider direction by executing different gestures. Widrow-Hoff Least Mean Squares (LMS) algorithm is used to train Single Layer Perceptron (SLP) structure. Whereas, for the training of Multi-Layer Perceptron (MLP) structure, a Back-Propagation (BP) algorithm is applied. Feature vector is created with AutoRegression (AR) model parameters. The classification accuracy achieved was $95 \%$. However the system requires a more robust classifier for the utilization of both biceps and triceps data to accommodate users with disabilities who are unable to perform such clearly defined tasks as studied at the present time. Rosenberg (1998), came up with a similar type of work known as Biofeedback Pointer. This is a system of EMG controlled two-dimensional pointer. Moving direction of pointer is determined by the movement of wrist. The driving force of pointer is the classified EMG signals from four of the muscles used to move the wrist. The EMG signals are interpreted using neural network and the network needs to be trained for each user. The Biofeedback Pointer's simple neural network is computationally inexpensive. Although the use of simple network reduces accuracy but it is compensated by using four EMG sensors. No special hardware required to train the device. The training for every user is performed by following the pointer's motion on the screen. The main drawback with the current training is that the user's motions may not adequately synchronize with the cursor which may lead to poor classification accuracy.

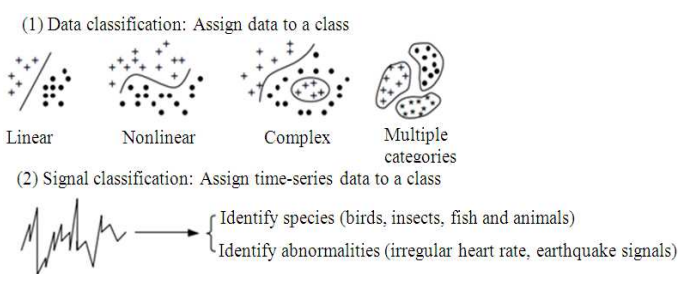

Fig. 1: Complex classification tasks of neural network (Jain et al., 1996) 


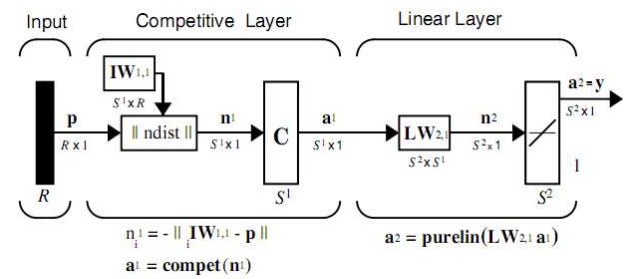

Fig. 2: LVQ network architecture (Kyung et al., 2007)

Kyung et al. (2007) have approached with another type of neural network based classifiers that would help to implement the HCI. They proposed a method of pattern recognition of EMG signals of hand gesture using spectral estimation and neural network. Their system comprises the Yule-Walker algorithm and the Learning Vector Quantization (LVQ). The Power Spectral Density (PSD) of the EMG signals is estimated by the Yule-Walker algorithm. In LVQ method, training of competitive layers has been done by supervised way. The learning of classifying input vectors is automatic for a competitive layer. The competitive layer finds the distance between input vectors which intern define the classes. However, the prominent drawback is that for similar input vectors, the competitive layer will put them in the same class. And so, from a strictly competitive layer design it is difficult to say whether or not any two input vectors are in the same class or in different classes. The experiment verified that EMG signals produced by hand gestures are reliably classified by the proposed system with a success rate of about $78 \%$. Figure 2 shows the LVQ network architecture used by Kyung et al. (2007).

EMG based mouse cursor control system as a manmachine interface was developed by Itou et al. (2001). They used Back-Propagation Neural Network (BPNN) algorithm with three inputs, two hidden layer and one output layer which achieved $70 \%$ rate of recognition. For their system, any muscle can be used and mouse cursor can be operated using a leg too. However, muscle fatigue may appear for long time use. To overcome the drawback of standard Artificial Neural Network (ANN), the muscle activity separation from different muscles in forearm was done by Naik et al. (2006). They utilized Independent Component Analysis (ICA) which is an iterative Blind Source Separation (BSS) technique that has been found to be very successful in audio and biosignal applications. Furthermore, Naik et al. (2007), came with improved ICA based BPNN algorithm. They focused on the input hidden context units, which give feedback to the hidden layer. Thus it gives the network an ability of extracting features of the data from the training events. The data is divided into subsets of training data (half of the data), validation (one-fourth of the data) and test subsets (onefourth of the data). The four Root Mean Square (RMS)
EMG values were the inputs and the outputs were the different isometric hand action RMS values. The overall accuracy was reported $97 \%$, but the number of hand gesture identification is restricted to three. There is also another further improvement for the identification of various hand gestures from same group of researchers. Naik et al. (2008), the researchers introduced multi run ICA of sEMG with BP learning algorithm based ANN classifier. Regarding their investigation it has been found that only ICA is not suitable for sEMG due to the nature of signal distribution and order ambiguity. It was reported that the classification of sEMG recording was almost in real-time with the utilization of combined mixing matrix and network weights. Their results indicate an overall classification accuracy of $99 \%$ for all the experiments. They pointed out that, the multi run ICA based BPNN can be used for to classify different subtle hand gestures. However, BPN cannot realize high learning and discrimination performance. The reason behind this is the EMG patterns, which differ considerably at the start and end of the motion even though they are within the same class. Whereas, El-Daydamony et al. (2008), applied HMM algorithm on sEMG that facilitates automatic sEMG feature extraction and ANN are combined for providing an integrated system for the automatic analysis and diagnosis of neuro-muscle disorders. The ANN architectures with three layers (input layer, hidden layer and output layer) were used by them. The ANN architectures are expressed as strings showing the number of inputs, the number of nodes in the hidden layers and two output nodes. They achieved the best correct classification rate and it was $90.91 \%$ for 80 hidden layers.

Fukuda et al. (1999) presented an EMG controlled pointing device using a neural network and developed a prototype system. Their proposed method is based on Log-Linearized Gaussian Mixture Network (LLGMN) introduced by Tsuji et al. (1995). In the proposed method, a several numbers of base directions are set on the computer display and the operator's intended direction is estimated from the probability that the pointer will move to each base direction. The neural network used to estimate the probability of the pointer movement and its velocity to each base direction. The structure of the proposed model is shown in Fig. 3. The accuracy improvement depends on the increased number of the base directions. However, much longer learning time required for a large number of the base directions. Additionally, the error becomes large if the desired direction differs from the base direction. Although this method can control the pointer in an arbitrary direction, but accuracy of the estimated direction is not high enough to the intention of the operator. 


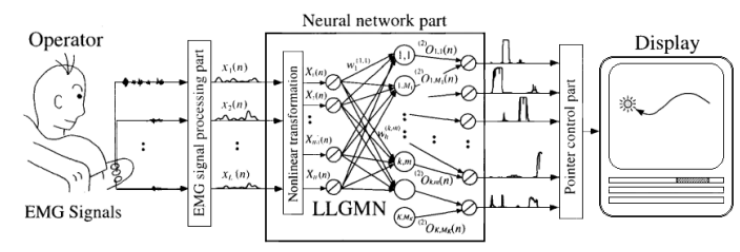

Fig. 3: The structure of the prototype system (Fukuda et al., 1999)

Furthermore, the number of moving directions will be infinite if the pointer is allowed to move in all directions from the current position. Tsuji et al. (2003) has therefore come with improved proposal to overcome the mentioned drawbacks. They proposed a Recurrent Log-Linearized Gaussian Mixture Network (R-LLGMN) based on Continuous Density Hidden Markov Model (CDHMM) (Xiang et al., 2007a). This network uses recurrent connections added to the units of LLGMN to discriminate a time sequence of the signals with higher accuracy. Based on Tsuji et al. (2003) modified theory, Fukuda et al. (2003) developed a new EMG-controlled omni-directional pointing device. A combination of finite base directions used in the proposed pointing device to represent an arbitrary direction of pointer movements. Use of neural network helps to avoid heavy learning calculation and a huge network structure by estimating the probability for each base direction. The probability of pointer movements in each base direction is estimated by R-LLGMN based on probability theory. Similar type of network has been utilized by Bu et al. (2004). According to their proposed system, a Probabilistic Neural Network (PNN) provides a stochastic perspective of pattern discrimination which is efficient for complicated data such as bioelectric signals. This PNN called a LLGMN, which estimates the posterior probability based on a Gaussian mixture model and the log-linear model. They have done hardware modeling of PNN using Field-Programmable Gate Array (FPGA), which is a System on Chip (SoC) design of a bioelectric human interface device.

The LLGMN has been successfully utilized to pattern discrimination of bioelectric signals, e.g., EMG and EEG. This network has been further used to develop various human interface applications like prosthetic device control, an EMG-based pointing device (Liyu et al., 1999). The difficulty includes non-trivial in cases of implementation of larger and more complicated neural networks and therefore more hardware efficient algorithms are required.

Other methodologies: Wheeler and Jorgensen (2003) and Wheeler (2003) approached with the design and implementation of neuroelectric interfaces for the controlling of virtual devices. EMG signals from Hand gestures are used to interface with a computer instead of manipulating mechanical devices such as joysticks and keyboards. The pattern recognition method employed was a HMM. They have selected moving average as feature because of its simplicity. For feature space, they have found that moving average is the best comparing with other common methods like Short Time Fourier Transform (STFT), Wavelets, AR coefficients. The ability to naturally interface with a computer which in terns allow human to manipulate any electrically controlled mechanical system. Beside wearable computing applications, the interface can also be applied in robotic arms, robotic exoskeletons, mobile robots for urban rescue, unmanned aircraft drones and space suit. There are side benefits of using EMG signals for control in long duration space missions. However, living in a zero gravity environment for extended periods may raise the muscle atrophy. Furthermore, recognition performance may greatly degraded by the unintentional misplacement of wet electrodes. Using of standard dry electrodes incorporated into a sleeve alleviated this problem but the signal sensing reliability then reduced.

Several researchers introduced linear classifiers for the classification of EMG signals. In the year of 2000, Bayes theorem employed by Alsayegh (2000) for EMGbased human-machine interface system. This system interprets a arm gestures in the 3-D space. Gestures interpretation has been done by sensing the activities of three muscles, namely, anterior deltoid, medial deltoid and biceps brachii muscles. The problem of gesture classification is carried out in a framework of the statistical pattern recognition based on the framework of Bayes theorem. A particular gesture identified by the processing of the EMG signals which utilizes the temporal coordination activity of the monitored muscles. Successive feature vectors for each gesture are constructed from the classification procedure. The overall success rate reported in the research is $96 \%$. It is also found that the structured type movements have a higher classification success rate than the pointing movements. This is because of clear coordination of the muscular activities in structured type. However, the input method described there is of course non-standard, since it does not make use of a keyboard or a mouse. Additionally, it is inappropriate for helping disabled persons, since it still requires control over the hands. Xiang et al. (2007b) also came with a Byes based EMG classifier. They implemented multiple hand gesture recognition along with a 2-D accelerometer for mobile HCI. In their research, Mean Absolute Value (MAV), the ratio of two MAVs and fourth-order AR model coefficients are used as feature vectors. The training of Linear Bayesian Classifier has done with the feature vectors to distinguish the different gesture actions from each other. The classical linear classifiers are well suited for real-time gesture analysis and real life implementation because of their low computational 
complexity and stable recognition performance. It has been mentioned that the combination of accelerometers and sEMG sensors provide higher classification accuracy. It was reported that classification is higher for the gesture sets including wrist motions, than the approaches made using only the accelerometers or SEMG sensors. Another type of research work based on linear classifier has been done by Kim et al. (2008). They have developed an EMG based interface system for hand gesture recognition. For realizing real-time classification with acceptable recognition accuracy, they represented the combination of two simple linear classifiers (K-Nearest Neighbor (KNN) and Bayes) in decision level fusion. The proposed EMG-based controlling interface is able to reliably recognize various hand gestures. In contrast to related work which is based on multiple EMG sensors, their proposed method gives a positive classification rate of over $94 \%$ even though only one EMG sensor is used. Moreover, since the EMG signal can be used to sense isometric muscular activity, it is possible to detect motionless gesture or intention in the EMG signal. Consequently, there is a wide range of potential applications using EMG signal in human-machine interfacing. However, to realize advanced applications, many issues still need to be resolved, including the development of algorithms for EMG-specific analysis, the extraction of relevant features and the design of real-time classifiers with guaranteed accuracy.

EMG classification methodologies for prosthetics: Most of the research work in this area has focused on using the muscles of the upper arms and/or shoulder muscles for the controlling of gross orientation and grasp of a low-degree-of-freedom prosthetic devices to manipulate objects. For more than three decades, researchers have been working on using EMG as a means for amputees to use remaining muscles to control prosthetic limbs. In recent years, the researchers started to contribute for controlling multi-fingered prosthetic hand using forearm muscles in hand amputees. They have applied different kinds of mathematical models and pattern recognition techniques for the classification of EMG. However, fully functional prosthetic hand is still unavailable commercially and this is clearly a promising new area of EMG research. In the area of controlling prosthetic hand, several types of ANN and Fuzzy logics used by the researchers. Their achieved results have good performance for EMG pattern recognition. Some other methodologies like linear classifier (k-NN, Bayes), Support Vector Machine (SVM), HMM also applied by few researchers with satisfactory classification output.

Neural network: Cai et al. (1999) proposed three layered feed-forward Artificial Neural Network (ANN) with $90 \%$ average classification accuracy of forearm motion. They used wavelet coefficients as feature sets for the training of BPANN. They performed six levels of decomposition of the signal onto to wavelet basis using Mallat (1989) algorithm. From 2-channel signals of each motion, they extracted 12 parameters to get feature vectors. These feature vectors has been used to train the ANN with 30 hidden layers, 4 output nodes. It is reported that, good localization properties of wavelets both in frequency and time domains, greatly improved the performance of ANN classifier. Jiang et al. (2005) also applied wavelet based ANN for the identification finger flexing motion. Each of the 16-channel EMG signals are decomposed in two orthogonal sub-space and Sym4 wavelet function is used. The arithmetic and wavelet coefficients for multi-scale decomposed signals are obtained for corresponding feature space. The feature space used were variance, maximum and mean absolute value of each coefficients. The ANN has 3 layers feed with three features, 36 cells of middle layer and output layer of 6 cells to identify 6 finger motions. They used 50 sets of user data to train ANN using BP algorithm and other 50 sets used for testing. The presented result shows that the correct recognition rates above $80 \%$. They concluded that the proposed novel method is effective for the identification of finger flexing and it is an alternative approach to use the surface EMG in controlling the finger motion of a multi-fingered prosthetic hand.

Online classification of EMG signals has been carried out by Tsenov et al. (2006) using various neural networks methods. They also pointed out a comparison between different intelligent computational methodologies regarding classification. It has been discovered that the classification performance of hand and finger movements depends significantly upon feature extraction. For higher and accurate classification, it is very much important to chose relevant features. With visual presentation of time domain feature sets, the researchers found that every movements are clearly distinguishable. The extracted time domain features are: MAV, Variance, Waveform Length (WL), Norm, Number of Zero Crossings, Absolute Maximum, Absolute Minimum, Maximum minus Minimum and Median Value (Med). After comparison between different intelligent computational methods of identification, they achieved best classification result (nearly 93\% using 2-channel data) using MLP other than Radial Basis Function (RBF) or LVQ. Also it is reported that the classification accuracy improve slightly when four channels of data is used. The main key steps and algorithm goal of online classification of hand movements using EMG signals are shown in Fig. 4. They have concluded that selection of relevant features will lead to high and accurate classification rates. 


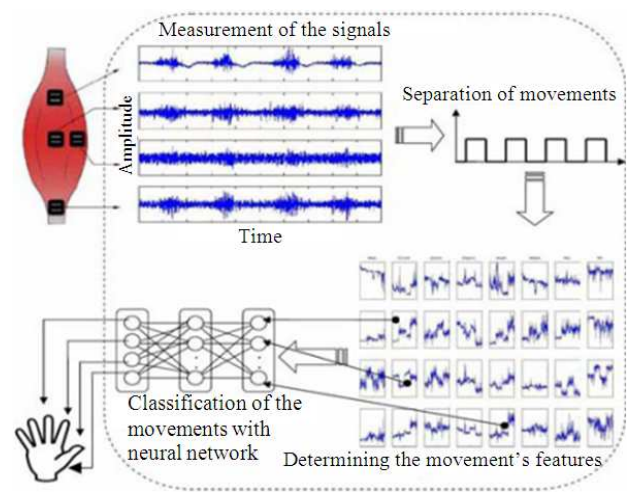

Fig. 4: The major steps of online classification of hand movements using EMG signals (Tsenov et al., 2006)

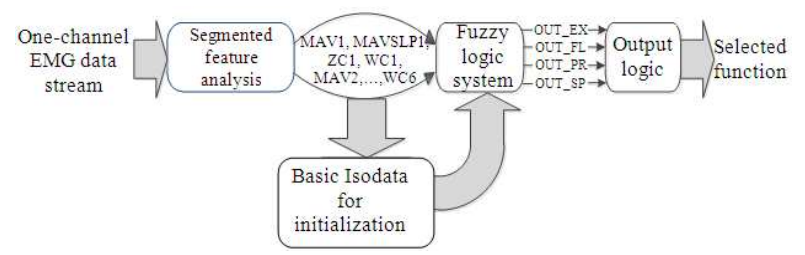

Fig. 5: Schematic diagram of fuzzy approach for EMG classification (Yang et al., 1996)

Furthermore, it is required to work with hardware realization and amputees for real-life implementation. However, in practice, determination of relevant features is very difficult. Additionally, for the online classification of hand movements, a well trained network structure needs to prepare from offline and double amount processing power required for two channels of data.

Another promising novel research work has been done by Farid and Keyvan (2006). They focused on the online estimation of a time-varying dynamic model of human arm for contact applications where the motion of the arm is limited to elbow movement in the horizontal plane. The proposed method uses Moving Window Least Squares (MWLS) to locally identify dynamic parameters for a limited number of operating points in a variable space defined by elbow joint angle and velocity and the EMG signals collected from upper-arm muscles. The model parameters are identified for a limited number of points using a MWLS estimation method. As in contact applications, the arm workspace and movement is relatively small and slow, the limited number of points is being justified. A Radial Basis Function Artificial Neural Network (RBFANN) is then trained with the limited points. Using upper arm EMG signals and elbow joint angle and angular velocity, this trained network is then utilized for online interpolation/extrapolation of the quasi-linear dynamic model parameters for other operating points. It is found that the online identified parameters clearly follow the pattern of changes in arm impedance. The level of accuracy obtained is enough for arm dynamic analysis and improved tradeoff between stability and performance in tele-robotic and haptic control systems. As pointed by them that the major factor in parameter error is due to the stochastic nature of EMG signals. It is also found that the online estimation accuracy may be improved by changing the neural network input quantization level and the use of more sensors for each muscle for more accurate representation of muscle activation levels. One specified drawback is that the network generated for each subject is not applicable to other subjects (inter-subject) and a separate network needs to be trained for each individual.

Fuzzy logic: Although biomedical signals are not always strictly repeatable and may sometimes even be contradictory; using fuzzy logic systems are advantageous. One of the most prominent features is that contradictions in the data can be tolerated in fuzzy logic system. In addition to that, using trainable fuzzy systems, it is possible to discover patterns in data which are not easily detected by other methods, similar to neural network. It is possible to integrate this incomplete but valuable knowledge into the fuzzy logic system due to the system's reasoning style as like as human being. This is a significant advantage over the ANN.

Yang et al. (1996) and Chan et al. (2000) proposed a new fuzzy approach for EMG recognition based on most of the same time-segmented features as used by Hudgins and Parker (1993); the results are compared to those of the ANN method. The schematic diagram of the method is shown in Fig. 5. They also presented a comparison of approached fuzzy method with an ANN method on four subjects and obtained very similar classification results. The EMG signals are divided into several time segments to preserve pattern structure and features were extracted from these segments. The features include MAV, Mean Absolute Value Slope (MAVSLP), number of Zero-Crossings (ZC), Slope Sign Changes (SSC) and wave length or Wave Complexity (WC) (Hudgins and Parker, 1993). The time segmented features is then feed to a fuzzy system for training and classification. To achieve acceptable training speed and realistic fuzzy system structure, these features are clustered without supervision using the Basic Isodata algorithm at the beginning of the training phase. Afterwards, the clustering results are used in initializing the fuzzy system parameters and were trained with BP algorithm. The given comparison and results proves that training process and generalization capability turned out to be superior than the ANN. The main advantages of the fuzzy system are it gives more consistent classification results and 
insensitive to over-training. Besides, when applied to pattern recognition problem, the threshold is more flexible than SIG-ANN. More favorably, it can incorporate expert experience easily by adding fuzzy units in parallel.

Park and Lee (1998) also come with EMG pattern recognition method for more accurate identification of a motion command at the same year 1998. Their proposed method based on AI (Winston, 1992) is able to accommodate expected interindividual differences and requires little computing time in the pattern recognition with the extracted feature parameters. The feature parameters used based on some of previous research; namely integral absolute value, difference absolute mean value, variance, Autoregressive (AR) model coefficients and linear cepstrum coefficients. In addition, they extracted the non-stationary property (Shwedyk et al., 1977) of EMG signals and the adaptive cepstrum vector (Lee et al., 1996) as feature parameter. The schematic of their proposed method is shown in Fig. 6. The Dempster-Shafer theory of evidence (Yager, 1987; Zeigler, 1988) is employed as an evidence accumulation method for the pattern recognition. A fuzzy mapping function has been designed for the application of the Dempster-Shafer theory of evidence and a series of evidence accumulation procedures according to each motion used. They evaluated that the proposed method recognized the desired motion efficiently with the multiple incomplete feature parameters. Also, the recognition error rates show that the proposed recognition method is superior to the other methods for EMG pattern recognition. The advantages of this approach to EMG pattern recognition are generating reasonably accurate results with less computing time and little subject training.

The method proposed by Weir and Ajiboye (2003) provide a new multifunctional prosthetic hand mechanism interfaced to the user using a four site Myoelectric (EMG) controller based on fuzzy-logic techniques. They stated that the fuzzy logic was explored as a method of discriminating between multiple surface EMG control signals because fuzzy logic lends itself to systems when there is a human component to be considered. A simple amplitudedriven inference rule base, constructed using Fuzzy CMeans (FCMs), achieved real-time classification rates from $94-99 \%$, during both steady state motions and transitions between movements. Rule generation performed automatically using the technique of fuzzy clustering (Altrock, 1995; Ross, 1995; Ajiboye and Weir, 2005). The presented result shows that higher classification accuracy is achieved and it is possible for the user to effect different control motions to control different functions. However, amputees did not participate in real-time testing and the possibility that they might have been more prescient at generating other "atypical" movements was not explored, only sequential control can be performed other than parallel control.

ICA is a new technique to separate blind sources, which has been used in some challenging fields of signal processing. For example, ICA could provide solutions to de-noise EMG, EEG or functional Magnetic Resonance Imaging (fMRI) signals for medical applications. Guangying (2007) introduced myoelectric prostheses hand based on ICA and Fuzzy controller. The researcher used FastICA algorithm which based on maximum negentropy and successfully decomposed sEMG signals. As presented, the pattern recognition rate obtained $85 \%$ and the fuzzy controller has the properties of reliability and adaptability. However, the major drawback is that if the numbers of source signal are unknown, some time it is larger than the sensor signals, then the blind separate problem becomes more difficult. The experimental procedure by (Guangying, 2007) is shown in Fig. 7.

In 2007, a novel approach introduced by Khezri and Jahed (2007) for the recognition of hand movements via sEMG patterns. For pattern recognition of sEMG signals for various hand movements, they utilized two intelligent classifiers: ANN and Fuzzy Inference System (FIS) were utilized. Figure 8 shows the brief overview of the techniques implemented for the proposed system. During the feature extraction steps, sEMG signal is processed with respect to three types of feature representations-Time Domain (TD), Time-Frequency domain (TFR) and their combination.

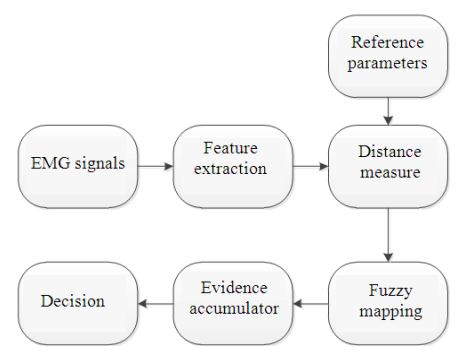

Fig. 6: Schematic diagram (Park and Lee, 1998)

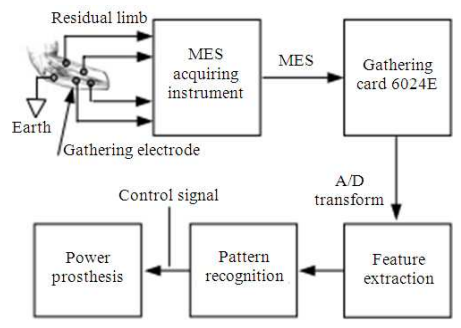

Fig. 7: The procedure of experimental system (Guangying, 2007) 


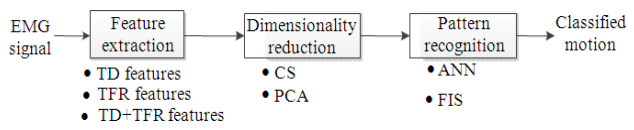

Fig. 8: Overall scheme of sEMG pattern recognition system (Khezri and Jahed, 2007)

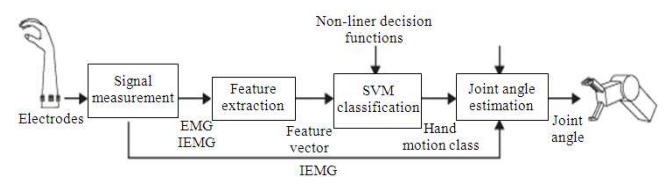

Fig. 9: Configuration of proposed method (Yoshikawa et al., 2006)

Next Class Separability (CS) and Principle Component Analysis (PCA) technique applied for the purpose of dimensionality reduction to simplify the task of the classifier. Results demonstrated that using the combined feature sets along with PCA as dimensionality reduction technique and FIS as classifier, best performance was achieved.

Other methodologies: An intelligent approach based on genetic algorithms, fuzzy measure and domain knowledge on pattern recognition, namely Supervised Feature Mining (SFM) method proposed by Huang et al. (2003). Their focus was on the selection of feature subset with the best discrimination ability from those features for classifying EMG signals. They selected eight kinds of different features that have been widely used to classify EMG signals as the feature candidates. They are Integral of EMG (IEMG), WL, variance, ZC, SSC, Willison Amplitude (WAMP) (Mahyar et al., 1995), Autoregressive Model (ARM) and Histogram of EMG (HEMG) (Mahyar et al., 1995), respectively. Accordingly, k-NN used for the classification of eight types of frequently prehensile postures. They reported that the classification rates of all postures are above $80 \%$. As a part of their work, they also found that the features ARM and HEMG have higher average classification rates than others. In 2005, HMM-based approach with higher classification accuracy presented by Chan and Englehart (2005). HMM method has the inherent ability to deal with spurious mis-classification. Even without majority vote, to perform as well as the MLP method with majority vote. Although there is an increase in computational load associated with adding majority vote to the HMM method, it use may still be justified. HMM classification of continuous myoelectric signals resulted in an average accuracy of $94.63 \%$, which exceeds their previous MLP method used in (Englehart et al., 2003) (93.27\%). This HMM classification methodology is also considerably simpler than the hybrid HMM and MLP, with genetic algorithm, method use in (Kwon et al., 1998) and has a higher accuracy (87.7\%). The largest gain in accuracy will be obtained when the user is provided with visual feedback. Yoshikawa et al. (2006) are out of few researches who has been applied SVMs to EMG signal classification for estimating operator's hand motions. They introduced a real-time hand motion estimation method using the EMG signals with the SVMs. Figure 9 shows the configuration of their proposed system. They have used cepstrum coefficients as frequency features in combination with the amplitude of EMG. They analyzed that the cepstrum coefficient feature improved the classification performance better than the temporal features such as the regression coefficients. Their result showed that the average classification accuracy is more than $90 \%$, however they didn't carry out online experiments of the proposed method.

\section{CONCLUSION}

EMG signal collected form surface of the skin has been used in diverse applications. HCI and prosthetic hands based on EMG signal are useful for habilitation or rehabilitation of persons with movement impairments. As the quantity of information dealt with by human operators is rapidly increasing with the development of information technology, so now it is a demand from the society to develop alternative interface for the old and disabled people. This study reviewed on two broad domain of EMG signal application namely: HCI and Prosthetic. Different EMG classification methodologies/techniques along with their applications as well as advantages and drawbacks have been discussed wherever it was found. This revision clearly pointed out the EMG signals classifications methodologies which will help the researchers' to find efficient methods. There are still huge possible ways to work for the ever-increasing number of persons with movement impairments, chronic disabled and older people by developing new methods, more natural more reliable interface which will improve QOL of them.

\section{REFERENCES}

Ajiboye, A.B. and R.F. Weir, 2005. Fuzzy C-means clustering analysis of the EMG patterns of six major hand grasps. Proceedings of Rehabilitation Robotics, June 28-July 1, IEEE Press, Chicago, IL., pp: 49-52. DOI: 10.1109/ICORR.2005.1501049

Alsayegh, O.A., 2000. EMG-based human-machine interface system. Proceedings of the IEEE International Conference on Multimedia and Expo, ICME 2000, July 30-Aug. 2, IEEE Press, New York, pp: 925-928. DOI: 10.1109/ICME.2000.871510

Altrock, C.V., 1995. Fuzzy Logic and Neuro Fuzzy Applications Explained. Prentice Hall PTR, New Jersey, ISBN: 978-0133684650, pp: 368. 
Bu, N., T. Hamamoto, T. Tsuji and O. Fukuda, 2004. FPGA implementation of a probabilistic neural network for a bioelectric human interface. Proceedings of the 47th Midwest Symposium on Circuits and Systems, July 25-28, IEEE Press, Hiroshima, Japan, pp: 29-32. DOI: 10.1109/MWSCAS.2004.1354283

Cai, L., Z. Wang and H. Zhang, 1999. An EMG classification method based on wavelet transform. Proceedings of the 21st Joint BMESEMBS Conference, (BMESEMBS'99), IEEE Press, Atlanta, GA., pp: 565. DOI: 10.1109/IEMBS.1999.802643

Chan, D.C. and K.B. Englehart, 2005. Continuous myoelectric control for powered prostheses using hidden Markov models. IEEE Trans. Biomed. Eng., 52: 121. DOI: 10.1109/TBME.2004.836492

Chan, F.H.Y., Y.S. Yang, F.K. Lam, Y.T. Zhang and P.A. Parker, 2000. Fuzzy EMG classification for prosthesis control. IEEE Trans. Rehabil. Eng., 8: 305-311. DOI: 10.1109/86.867872

El-Daydamony, E.M., M.E. Gayar and F.A. Chadi, 2008. A computerized system for SEMG signals analysis and classification. Proceeding of the National Radio Science Conference, Mar. 18-20, IEEE Press, Tanta, Egypt, pp: 1-7. DOI: 10.1109/NRSC.2008.4542388

Englehart, K., B. Hudgins and A.D.C. Chan, 2003. Continuous multifunction myoelectric control using pattern recognition. Technol. Disability, 15: 95-103.

Farid, M. and H.Z. Keyvan, 2006. A Method for online estimation of human arm dynamics. Proceedings of the 28th Annual International Conference of the IEEE Engineering in Medicine and Biology Society, Aug. 30-Sept. 3, IEEE Press, New York, pp: 2412-2416. DOI: 10.1109/IEMBS.2006.260332

Fukuda, O., J. Arita and T. Tsuji, 2003. An EMGcontrolled omnidirectional pointing device using a HMM-based neural network. Proceedings of the International Joint Conference on Neural Networks, July 20-24, IEEE Press, USA., pp: 3195-3200. DOI: $10.1109 /$ IJCNN.2003.1224084

Fukuda, O., T. Tsuji and M. Kaneko, 1999. An EMG controlled pointing device using a neural network Systems. Proceedings of the IEEE International Conference on Man and Cybernetics, Oct. 12-15, IEEE Press, Tokyo, Japan, pp: 63-68. DOI: 10.1109/ICSMC.1999.812377

Guangying, Y., 2007. Study of myoelectric prostheses hand based on independent component analysis and fuzzy controller. Proceedings of the 8th International Conference on Electronic Measurement and Instruments, Aug. 16-July 18, IEEE Press, Xian, China, pp: 1-174-1-178. DOI: 10.1109/ICEMI.2007.4350416

Huang, H.P., Y.H. Liu and C.S. Wong, 2003. Automatic EMG feature evaluation for controlling a prosthetic hand using a supervised feature mining method: An intelligent approach. Proceedings of the IEEE International Conference on Robotics Automatics, Sept. 10-15, IEEE Press, Taipei, Taiwan, pp: 220-225.
Hudgins, B. and P.A. Parker, 1993. A new strategy for multifunction myoelectric control. IEEE Trans. Biomed. Eng., 40: 82-94. DOI: 10.1109/10.204774

Itou, T., M. Terao, J. Nagata and M. Yoshida, 2001. Mouse cursor control system using EMG. Proceedings of the 23rd IEEE Annual International Conference of the IEEE Engineering in Medicine and Biology Society, Oct. 25-28, IEEE Press, Istanbul, Turkey, pp: 1368-1369.

Jain, A.K., J. Mao and K.M. Mohiuddin, 1996. Artificial neural networks: A tutorial. IEEE Comput., 29: 31-44. DOI: 10.1109/2.485891

Jiang, M.W., R.C. Wang, J.Z. Wang and D.W. Jin, 2005. A method of recognizing finger motion using wavelet transform of surface EMG signal. Proceedings of the International Conference of the IEEE EMBS, Jan. 1718, IEEE Press, Shanghai, China, pp: 2672-2674. DOI: 10.1109/IEMBS.2005.1617020

Khezri, M. and M. Jahed, 2007. A novel approach to recognize hand movements via sEMG patterns. Proceedings of the 29th Annual International Conference of the IEEE Engineering in Medicine and Biology Society, Aug. 22-26, IEEE Press, Lyon, France, pp: 4907-4910. DOI: 10.1109/IEMBS.2007.4353440

Kim, J., S. Mastnik and E. Andre, 2008. EMG-based hand gesture recognition for real-time biosignal interfacing. Proceedings of the 13th International Conference on Intelligent User Interfaces, Jan. 1316, IEEE Press, Gran Canaria, Spain, pp: 30-39. DOI: $10.1145 / 1378773.1378778$

Kwon, J., S. Lee, C. Shin, Y. Jang and S. Hong, 1998. Signal hybrid HMM-GA-MLP classifier for continuous EMG classification purpose. Proceedings of the 20th Annual International Conference IEEE EMBS, Oct. 29-Nov 1, IEEE Press, Hong Kong, pp: 1404-1407. DOI: 10.1109/IEMBS.1998.747145

Kyung, K.J., W.K. Joo, K.L. Hyun, B.C. Sung and H.E. Ki, 2007. EMG pattern classification using spectral estimation and neural network. Proceedings of the SICE, Annual Conference, Sept. 17-20, IEEE Press, Takamatsu, Japan, pp: 1108-1111. DOI: 10.1109/SICE.2007.4421150

Lee, S.P., J.S. Kim and S.H. Park, 1996. An enhanced feature extraction algorithm for EMG pattern classification. IEEE Trans. Rehab. Eng., 4: 439-443. DOI: $10.1109 / 86.547948$

Liyu, C., W. Zhizhong and Z. Haihong, 1999. An EMG classification method based on wavelet transform. Proceedings of the 21st Joint BMESEMBS Conference, Oct. 13-16, IEEE Press, Atlanta, GA., pp: 565. DOI: 10.1109/IEMBS.1999.802643

Mahyar, Z.K., W.C. Bruce, B. Kambir and M.H. Reza, 1995. EMG feature evaluation for movement control of upper extremity prostheses. IEEE Trans. Rehab. Eng., 3: 324-333. DOI: 10.1109/86.481972 
Mallat, S., 1989. A theory for multiresolution signal decomposition: the wavelet representation. IEEE Trans. Patt. Anal. Mach. Intell., 11: 664-693. DOI: 10.1109/34.192463

Naik, G.R., D.K. Kumar and H. Weghorn, 2007. Performance comparison of ICA algorithms for Isometric Hand gesture identification using Surface EMG intelligent sensors. Proceeding of the 3rd International Conference on Sensor Networks and Information, Dec. 3-6, IEEE Press, Melbourne, Australia, pp: 61-618. DOI: 10.1109/ISSNIP.2007.4496913

Naik, G.R., D.K. Kumar and M. Palaniswami, 2008. Multi run ICA and surface EMG based signal processing system for recognizing hand gestures. Proceeding of the 8th IEEE International Conference on Computer and Information Technology, July 8-11, IEEE Press, Sydney, Australia, pp: 700-705. DOI: 10.1109/CIT.2008.4594760

Naik, G.R., D.K. Kumar, V.P. Singh and M. Palaniswami, 2006. Hand gestures for HCI using ICA of EMG. Proceedings of the HCSNet Workshop on Use of Vision in Human-Computer Interaction, Nov. 01, Canberra, Australia, pp: 67-72.

National Spinal Cord Injury Association, 1996. Fact Sheets \#2: Spinal Cord Injury Statistics. http://users.erols.com/nscia/resource/factshts/fact02.html

Park, S.H. and S.P. Lee, 1998. EMG pattern recognition based on artificial intelligence techniques. IEEE Trans. Rehab. Eng., 6: 400-405. DOI: 10.1109/86.736154

Putnam, W. and R.B. Knapp, 1993. Real-time computer control using pattern recognition of the electromyogram. Proceedings of the 15th Annual International Conference of the IEEE Engineering in Medicine and Biology Society, IEEE Press, San Diego, USA., pp: 1236-1237.

Rosenberg, R., 1998. The biofeedback pointer: EMG control of a two dimensional pointer. Proceeding of the 2nd International Symposium on Wearable Computers, Oct. 19-20, IEEE Press, Pittsburgh, PA., pp: 162-163. DOI: 10.1109/ISWC.1998.729546

Ross, T.J., 1995. Fuzzy Logic with Engineering Applications. 2nd Edn., John Willey and Sons, ISBN: 978-0-470-86075-5, pp: 650.

Shwedyk, E., R. Balasubraminian and R.N. Scott, 1977. A nonstationary model for the electromyogram. IEEE Trans. Biomed. Eng., 24: 417-424. DOI: 10.1109/TBME.1977.326175

Tsenov, G., A.H. Zeghbib, F. Palis, N. Shoylev and V. Mladenov, 2006. Neural networks for online classification of hand and finger movements using surface EMG signals. Proceedings of the 8th Seminar on Neural Network Applications in Electrical Engineering, Sept. 25-27, IEEE Press, Belgrade, Serbia and Montenegro, pp: 167-171. DOI: 10.1109/NEUREL.2006.341203

Tsuji, T., H. Ichinobe, O. Fukuda and M. Kaneko, 1995. A maximum likelihood neural network based on a loglinearized Gaussian mixture model. Proceedings of IEEE International Conference on Neural Networks, Nov. 27-Dec. 1, IEEE Press, Perth, WA., pp: 24792484. DOI: $10.1109 /$ ICNN.1995.487343
Tsuji, T., N. Bu, O. Fukuda and M. Kaneko, 2003. A Recurrent log-linearized Gaussian mixture network. IEEE Trans. Neural Network, 14: 304-316. DOI: 10.1109/TNN.2003.809403

Weir, R.F. and A.B. Ajiboye, 2003. A multifunction prosthesis controller based on fuzzy-logic techniques. Proceedings of the 25th Annual International Conference of EMBS, Sept. 17-21, IEEE Press, Mexico, pp: 1678-1681. DOI: 10.1109/IEMBS.2003.1279711

Wheeler, K.R., 2003. Device control using gestures sensed from EMG. Proceedings of the 2003 IEEE International Workshop on Soft Computing in Industrial Applications, June 23-25, IEEE Press, USA., pp: 21-26. DOI: 10.1109/SMCIA.2003.1231338

Wheeler, K.R. and C.C. Jorgensen, 2003. Gestures as input: Neuroelectric joysticks and keyboards. Pervasive Comput., IEEE., 2: 56-61.

Wiener, N., 1948. Cybernetics. 1st Edn., MIT Press, ISBN: 0-262-73009, pp: 116-129.

Winston, P.H., 1992. Artificial Intelligence. 3rd Edn., Addison Wesley, Reading, ISBN: 10: 9780201533774, pp: 750.

Xiang, C., Z. Xu, Z. Zhang-Yan, Y. Ji-Hai and V. Lantz et al., 2007a. Multiple hand gesture recognition based on surface EMG signal. Proceeding of the 1st International Conference on Bioinformatics and Biomedical Engineering, July 6-8, IEEE Press, Wuhan, China, pp: 506-509. DOI: 10.1109/ICBBE.2007.133

Xiang, C., Z. Xu, Z. Zhang-Yan, Y. Ji-Hai and V. Lantz et al., 2007b. Hand gesture recognition research based on surface EMG sensors and 2D-accelerometers. Proceedings of the 11th IEEE International Symposium on Wearable Computers, Boston, MA., Oct. 11-13, IEEE Press, pp: 11-14. DOI: 10.1109/ISWC.2007.4373769

Yager, R.R., 1987. On the Dempster-Shafer framework and new combination rules. Inform. Sci.: Int. J., 41: 93-137. DOI: 10.1016/0020-0255(87)90007-7

Yang, Y.S., F.K. Lam, F.H.Y. Chan, Y.T. Zhang and P.A. Parker, 1996. A new fuzzy approach for pattern recognition with application to EMG classification. Proceedings of the IEEE International Conference on Neural Networks, June 3-6, IEEE Press, Washington DC., pp: 1109-1114. DOI: 10.1109/ICNN.1996.549053

Yoshikawa, M., M. Mikawa and K. Tanaka, 2006. Real-time hand motion estimation using EMG signals with support vector machines. Proceedings of the International Joint Conference SICE-ICASE, Oct. 18-21, IEEE Press, Busan, South Korea, pp: 593-598. DOI: 10.1109/SICE.2006.315553

Zeigler, B.P., 1988. Some properties of modified Dempster-Shafer operators in rule-based inference systems. Int. J. Gen. Syst., 14: 345-356. DOI: 10.1080/03081078808935024 